\title{
Computational study of the elastic properties of Rheum rhabarbarum tissues via surrogate models of tissue geometry
}

Tanvir R. Faisal ${ }^{\mathrm{a}}$, Nicolay Hristozov ${ }^{\mathrm{b}}$, Tamara L. Western ${ }^{\mathrm{b}}$, Alejandro D. Rey ${ }^{\mathrm{c}}$ and Damiano Pasini $^{\mathrm{a}, *}$

${ }^{a}$ Department of Mechanical Engineering, McGill University, 817 Sherbrooke St. W., Montreal, QC H3A 0C3, Canada

${ }^{b}$ Department of Biology, McGill University, 1205 Docteur Penfield, Montreal, QC H3A 1B1, Canada

${ }^{\mathrm{c}}$ Department of Chemical Engineering, McGill University, 3610 Rue University, Montreal,QC H3A 0C5, Canada

*Corresponding author. Address: Department of Mechanical Engineering, McGill University, 817 Sherbrooke St. West, Montréal, Québec, H3A 2K6, Canada, Phone: (514) 398-6295, Fax:

(514) 398-7365, Email: damiano.pasini@ mcgill.ca

\begin{abstract}
Plant petioles and stems are hierarchical cellular structures, displaying geometrical features defined at multiple length scales. One or more of the intermediate hierarchical levels consists of tissues, in which the cellular distribution is quasi-random, a factor that affects the elastic properties of the tissues. The current work focuses on the finite element analysis (FEA) of the constituent tissues of the plant Rheum rhabarbarum (rhubarb). The geometric model is generated via a recently introduced method: the finite edge centroidal Voronoi tessellation (FECVT), which is capable to capture the gradients of cellularity and diversified pattern of cellular materials, as opposed to current approaches in literature. The effective stiffness of the tissues is obtained by using an accurate numerical homogenization technique via detailed finite element analysis of the models of sub-regions of the tissues. As opposed to a large-scale representative volume element (RVE), statistical volume elements (SVE) are considered in this work to model tissue microstructures that are highly random. 2D finite element analyses demonstrate that the distribution of cells in collenchyma and parenchyma tissue make them stiffer in two different directions, while the overall effect of the combined tissues results in approximately equal stiffness in both directions. The rhubarb tissues, on the other hand, are more compliant than periodic and quasi-uniform random cellular materials by a factor of up to $47 \%$ and $44 \%$, respectively. The variations of the stiffness shows the stiffening role that cell shape, size, and graded cellular distribution play in the mechanics of the rhubarb tissue.
\end{abstract}

\section{Keywords}

FECVT; SVE; Finite Element Analysis; Collenchyma; Parenchyma 


\section{Introduction}

Plants are one of the major kingdoms in biology. From a structural point of view, plants and their organs frequently exhibit excellent mechanical properties. Among their organs, the petiole that attaches the leaf to the stem resembles a cantilever beam, which supports the leaf against gravity, allowing for its exposure to the sun. The petiole of Rheum rhabarbarum (rhubarb) plant as shown in Fig. 1(a) is an example of a cantilever beam that must resist combined loads including bending and twisting. The petiole's flexural and torsional stiffness are largely controlled by its overall geometric properties and the stiffness of its constituent tissues. A plant organ (e.g. petiole) is generally composed of an assembly of cellular tissues, which make up its microstructure and largely govern its physical properties. Each tissue grows to meet specific functional requirements that guarantee plant survival in a given environment. The way in which multiple tissues are geometrically assembled within an organ helps determine mechanical performance, important for structural support. It has been demonstrated that the shape, size, and spatial distribution of cells governs the physical, biological, and structural properties of a cellular (tissue) material (Ghosh et al., 1996; Pasini, 2008). The microstructural analysis of the virtually modelled cellular microstructure of a plant tissue is crucial to the understanding of its mechanical behaviour (Faisal et al., 2010). The results of a microstructural analysis, which might provide the stiffness of both the individual tissues and their combination help to provide insights into the effect of cell size, shape, and cellular distribution with clustered and complex area of higher gradients which usually occur in rhubarb petioles.

The petiole can be considered as a hierarchical structure, having structural features defined at multiple length scales. A petiole is generally composed of an assembly of cellular tissues, whose mechanical characteristics collectively depend on the geometry of their constituent cells, cell wall composition, the structural properties of the wall constituents, and the microarchitecture of the tissues. The orders of structural hierarchy considered in the current work for the rhubarb petiole are represented by $n$ as shown in Fig. 1. As indicated in Fig. 1, n=1 corresponds to the cell wall, $n=2$ to tissue, and $n=3$ to the petiole. This work specifically focuses on the microstructural analysis at the tissue level $(n=2)$ since the microstructural attributes of a tissue, such as the size and shape of the cells, and their distribution within a tissue largely affect the overall structural properties of the petiole. 


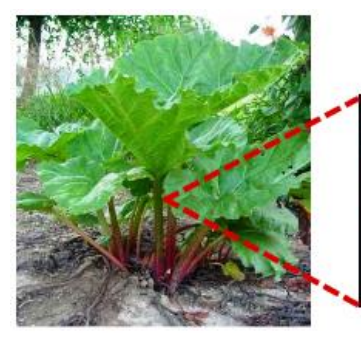

(a) Whole plant (b)

b) Petiole cross-section with multiple tissue $[\mathrm{n}=3]$

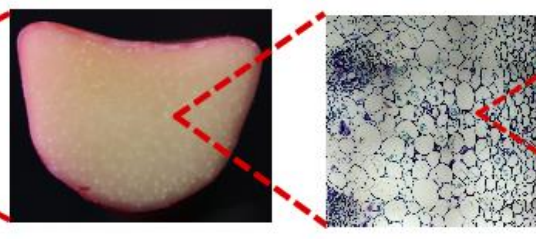

(c) Tissue microstructure $[\mathrm{n}=2]$

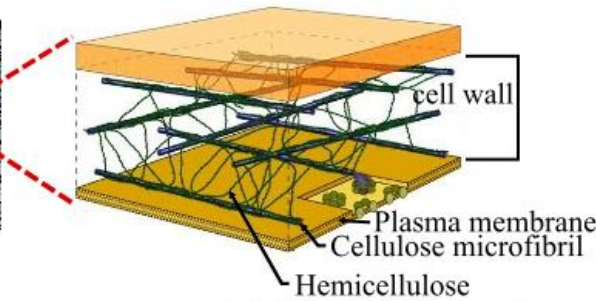

(d) Simplified model of cell wall ${ }^{2}$ $[\mathrm{n}=1]$

Fig.1. Multiscale hierarchical organization of a plant (Rheum rhabarbarum) petiole. [n] denotes the hierarchical level of the multiscale structure.

Microstructural analysis of cellular solids is crucial to understanding their overall behavior since cellular solids are prevalent both in nature and in human-made engineering structures. Many researchers have modeled natural cellular solids using repeating unit cells to construct a regular microstructure in the form of a circular, square or hexagonal array of cells (Gibson and Ashby, 1982). Closed-form relations of the structure-property can be derived with simplified geometric models based on repeated unit cells (Gibson and Ashby, 1999; Silva et al., 1995). Nevertheless, the modeling of plant tissue is a challenging task since natural cellular solids often exhibit non-periodic arrangement of cells. Since the microstructure of plant tissue is typically heterogeneous in shape and size, as shown in Fig. 2, the Voronoi tessellation can be used to generate an accurate representation of a non-periodic microstructure (Li et al., 2005; Silva and Gibson, 1997; Silva et al., 1995). The Voronoi tessellation is used extensively to model grain geometry for the property characterization of polycrystalline aggregates (Cailletaud et al., 2003) and inter granular cracks (Hussain et al., 1993). There is a strong correlation between the microstructural geometry and the structural properties of space-filling networks. The interdependence between topology, geometry and physical dynamics of the spherulitic grain size-shape arrangement in semi-crystalline polymeric cellular networks has been shown both experimentally and theoretically (Chan and Rey, 1997; Huang et al., 1999; Kamal et al., 1997). Mattea et al. (Mattea et al., 1989) and Roudot et al. (Roudot, 1990) pioneered the use of Voronoi tessellation to model the microstructure of fruit tissues. Both groups aimed only to capture the 
randomness of the fruit tissue microstructure without necessarily producing a model that accurately represented the real tissue. Recently, Ntenga et al. (Beakou and Ntenga, 2011; Ntenga and Beakou, 2011) tried to analyse the structure, morphology and mechanical properties of Rhectophyllum camerunense (RC) plant fiber using a conventional Voronoi diagram. Due to the inherent drawbacks of the Voronoi (CVT) model, semi-infinite edges were present at the boundary of the fiber, making the model unsuitable for Finite Element Analysis (FEA). To generate a geometric model, having finite edges at the boundary, a Finite-edge Centroidal Voronoi Tessellation (FECVT) method has been recently developed (Faisal et al., 2012). The FECVT method has been applied to model the tissue microstructures of Arabidopsis thaliana and Philodendron melinonii, two plants with distinct tissue architecture (Faisal et al., 2012). In this work, the FECVT method is applied to generate the virtual model of the rhubarb tissue (Fig. 2). In particular, the FECVT method is applied here for the first time to generate both the collenchyma and parenchyma tissues and their combined pattern, which is critical to determine the overall mechanical behaviour of rhubarb tissue. The presence of vascular bundles, clustered higher area gradients, in the parenchyma tissue, make the geometric replication of these patterned tissue challenging. This paper shows that the FECVT method can handle and replicate complex heterogeneity that emerges in plant tissues. Hence, the virtual models that will be obtained here for rhubarb allows calculate the effective stiffness of their tissue via FEA.

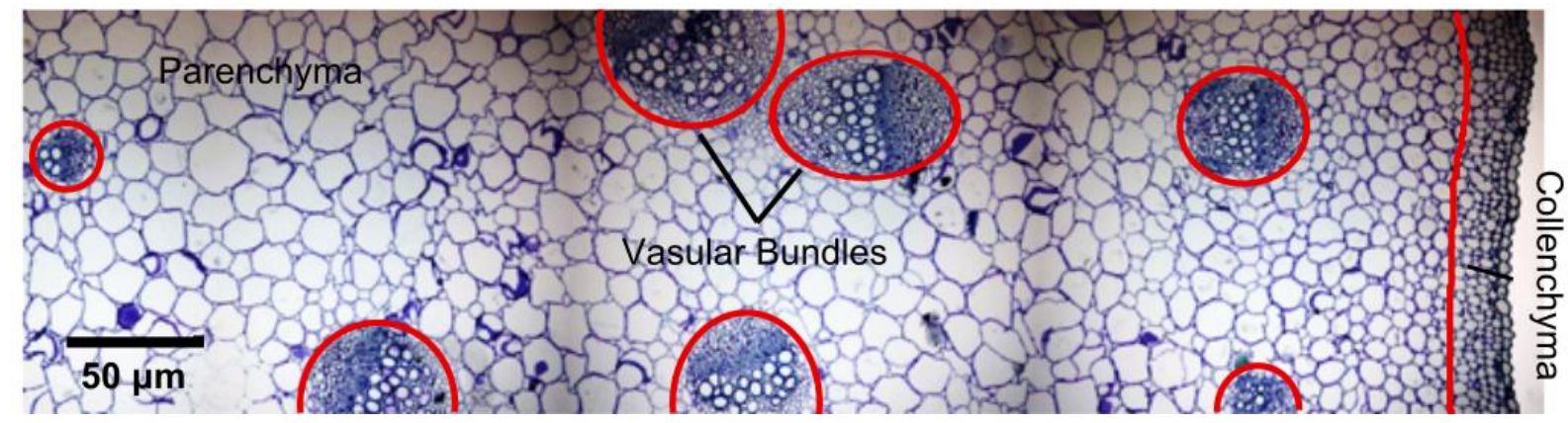

Fig.2. Paraffin-embedded rhubarb petiole cross-section stained with TBO and imaged with light microscopy at 20x magnifications. Approximately 16 photos were stitched together to create this composite image. Collenchyma cells, vascular bundle, and parenchyma cells are visible. Scale bar $=50 \mu \mathrm{m}$.

Most studies on the homogenization of cellular solids are based on regular models with a periodic microstructure. Real solid foams, however, exhibit amorphous arrangements of pores 
having different sizes and shapes far from being periodic. The homogenized/apparent elastic property for periodic honeycomb varies from 10 15\% compared to non-periodic honeycomb (Silva et al., 1995). To consider the microstructural irregularity, the Voronoi cell finite element method (VCFEM) was developed and coupled with asymptotic homogenization method to generate globally homogenized elastic properties (Ghosh et al., 1996). This method considers the local periodicity of the microstructure only. To take into account the global periodicity in the irregular microstructures represented by the Voronoi tessellation, the homogenization process requires a large scale representative volume element (RVE). Analyses of such models had been provided by, among others, Silva et al. (Silva et al., 1995), Fazekas et al. (Fazekas et al., 2002), Roberts and Garboczi (Roberts and Garboczi, 2001) for both two and three-dimensional models. These studies demonstrated that even a large scale RVE of a plant tissue having heterogeneous cellularity may not be representative of the respective tissue. Moreover, the large scale RVEs are inefficient in terms of computational effort. To overcome this limitation, the computational homogenization technique can be applied to several small scale RVEs, rather than to large scale RVE, with non-periodic microstructures for global homogenization. In this approach, the stochastically selected RVEs, termed as Statistical Volume Element (SVE) (Ostoja-Starzewski, 1993; Ostoja-Starzewski, 1998; Ostoja-Starzewski, 2006), are able to effectively consider the microstructural irregularity present in the plant tissues. For a given boundary condition, the effective mechanical properties of rhubarb tissues can be predicted by the numerical simulation of each SVE performed by finite element analysis. However, the concept of SVE is coupled with the FECVT method and for the first time applied here to determine the stiffness of plant tissue.

The specific objectives of this paper are to: 1) generate the geometric models of the constituent tissues of rhubarb petiole using FECVT algorithm; 2) determine the normalized effective (homogenized) stiffness of the constituent tissues based on finite element analysis of the SVEs (FECVT models); 3) compare the effect of alternative microarchitectures of the constituent rhubarb tissues with periodic and quasi-uniformly distributed cells.

The organization of this paper is as follows. Section 2 describes the methods adopted in this work to meet the objectives. Section 3 delineates the results obtained for the rhubarb tissues, and Section 4 interprets the physical significance of the results by comparing them with periodic and quasi-uniformly distributed microstructures. 


\section{Finite element modeling of the effective tissue stiffness}

Although the mechanical properties of a random microstructure can be determined by using direct numerical simulation, this strategy is computationally expensive to apply throughout the whole domain. Instead, the homogenized/effective properties of a material with random microstructure can be obtained from an RVE, which allows bypass the use of a large scale and detailed numerical simulation (Alber et al., 1992; Nemat-Nasser and Hori, 1999). The RVE contains the essential microstructural features and has been widely used to compute the effective material properties of heterogeneous and composite material having microstructural irregularities, such as grains, inclusions, voids, fibers and others. Since the intrinsic nonhomogeneity in the constituent tissues of the rhubarb petiole is highly random, a single FECVT model to capture the heterogeneity is not viable, because the requirement of the RVE size, being infinite, is neither practical nor desirable; furthermore, the simulation of a large RVE may suffer from a significant computational burden (Liu et al., 2010; Yin et al., 2008).

In the present analysis, to overcome the above limitations and to capture the effect of microstructural variability in the constitutive tissue properties, we adopt the concept of SVE. The size of the SVE is smaller than a conventional RVE, but larger than the microstructure characteristic length scale (Ostoja-Starzewski, 1993; Ostoja-Starzewski, 1998; OstojaStarzewski, 2006). Hence, each of the FECVT models of a particular tissue should correspond to the respective SVE for the numerical simulation so as to capture the microstructural randomness present in the tissue. To obtain the effective/homogenized mechanical properties, the numerical simulation of compressive deformation in each SVE is performed by finite element analysis. The stochastic approach of using several SVEs is, therefore, expected to better represent the overall randomness commonly appearing in a tissue.

\subsection{Geometric (virtual) modeling of plant tissue}

A Voronoi microstructure is constructed based on a set of randomly generated points called Voronoi sites. The cell boundaries are drawn such that any point within the enclosed polygon is closer to its Voronoi site than to the Voronoi sites of the surrounding polygons. The Voronoi tessellation thus divides a space into as many regions as the Voronoi sites. The centroids of the cells are here used to construct the Voronoi diagram, defining a centroidal Voronoi tessellation $(\mathrm{CVT})$. We operate as follows. First, a color or greyscale micrograph of a plant tissue is 
subjected to image segmentation. Thresholding, a method for image segmentation, is then used to convert the colour or greyscale image into a binary (black and white) image. The Canny edge detection algorithm (Canny, 1986) is used to detect the shapes of the cells precisely.

Once the shapes of the cells are detected, the $1^{\text {st }}$ order moments of the cells are computed using $X$ and $Y$ coordinates of the pixels. The algebraic form of the moment equation of a digital image is

$$
m_{p q}=\sum_{i=1}^{n_{1}} \sum_{j=1}^{n_{2}} X_{i}^{p} Y_{j}^{q} f(i, j)
$$

where $\left(X_{i}, Y_{j}\right)$ is the coordinate of the $i, j$ th pixel, $f(i, j)$ have value 1 if the $i, j$ th pixel is in the shape and 0 otherwise. Considering the region of interest, which is completely enclosed in a rectangular region $G$ of size $n_{1}$ by $n_{2}$ pixels, $i$ varies from 1 to $n_{1}$, and $j$ varies from 1 to $n_{2}$ in the function $f(i, j)$. For a $2 \mathrm{D}$ region, $p+q$ denotes the order of moment, where $p$ and $q$ are integers. Hence, the coordinates of a cell are

$$
\bar{X}=\frac{m_{10}}{m_{00}} \text { and } \bar{Y}=\frac{m_{01}}{m_{00}}
$$

where the zeroth moment, physically, is equal to the area of the region.

After determining the centroids of the cells, the Voronoi tessellation is constructed based on the Quick hull algorithm (Barber et al., 1996). The outcome is a conventional CVT with semiinfinite edges at the boundary. The finite element analysis of the Voronoi model having semiinfinite edges may lead to erroneous result (effective stiffness) since the boundary conditions applied at an infinite distance are not realistic. This problem is especially difficult to correct in models with irregular shape contour.

To remove the infinite edges from the boundary, the centroids of the outermost cells are determined. For each centroid, the distances between the centroid and the surrounding Voronoi sites (centroids of the surrounding polygons) are calculated, and the minimum distance is determined. An imaginary point is created such that the distance between itself and the centroid 
is half of the minimum distance. The imaginary point is thus created for each of the selected centroid. Based on the set of imaginary points and the convex hull algorithm (Barber et al., 1996), a boundary is imposed, after which a Boolean subtraction is realized. With this Boolean operation, the semi-infinite edges are truncated, and the vertices of the truncated edges are reconnected to form the final boundary. The FECVT technique is, therefore, capable of capturing the microstructure of an image with an arbitrarily shaped boundary contour. This computational algorithm, FECVT method (Faisal et al., 2012), is applied here to model the rhubarb tissue microstructure shown in Figs. 3 to 5. Since the micrographs are used to generate the geometric models of the tissues, the accuracy of the models appears to depend on the quality of the experimentally acquired micrographs. As a result, the cellular distribution of the surrogate model of the tissue strongly depends on the quality and image clarity of the micrographs. If the micrograph of the tissue microstructure is vivid and clear, the FECVT method can capture the details of a cellular distribution with a good level of accuracy.



Fig.3. FECVT model of collenchyma tissue of rhubarb petiole.
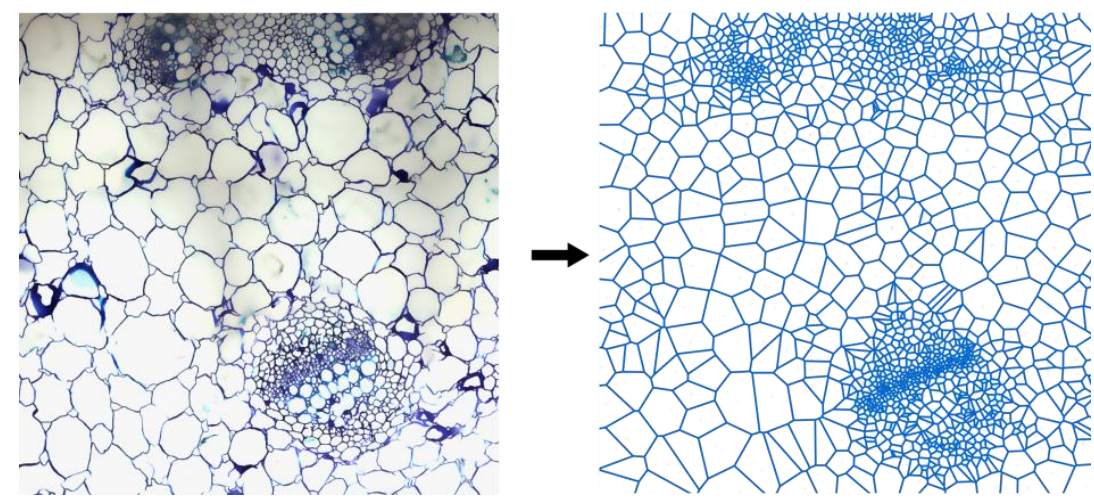

Fig.4. FECVT model of parenchyma tissue of rhubarb petiole. 


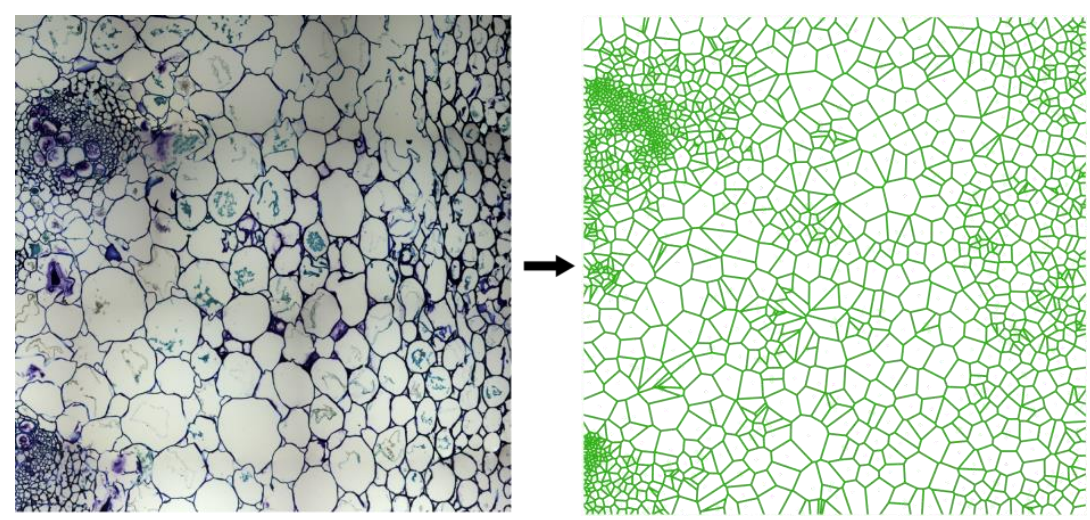

Fig.5. FECVT model of combined collenchyma-parenchyma (col-par) tissue of rhubarb petiole.

\subsection{Construction of the FE model}

The geometric information of the FECVT model (i.e., SVE) is transferred to ANSYS to generate the FEA model. The cell walls are considered to be straight and of uniform thickness throughout the surrogate tissue model. The relative density of a given model is specified by assigning the appropriate cell wall thickness. The constitutive behavior of the wall material is assumed to be elastic-perfectly plastic. Each cell wall of the Voronoi microstructure is modeled with a BEAM23 element, capable of describing both elastic and plastic behavior. The shear deformation, which is important for stubby beams, also is captured by considering the shear deflection coefficient of the beam element. The beam elements have a rectangular cross-section of uniform thickness $t$. The relative density, $\rho^{*} / \rho_{s}$ of the SVE, is given by

$$
\frac{\rho^{*}}{\rho_{s}}=\frac{\text { area of solid walls }}{\text { total area of Voronoi model }}=t \frac{\sum_{i=1}^{N} l_{i}}{L_{X} L_{Y}}
$$

where $N$ is the total number of beams, $l_{i}$ is the length of the beam $i ; L_{X}$ and $L_{Y}$ are the dimensions of the Voronoi model along the $X$ and $Y$ axes, respectively. The FEA is conducted for different relative densities, adjusted by the value of $t$. In the finite element analysis, a Young's modulus 
$E_{s}=1$ is assigned to each beam to obtain the normalized tissue stiffness. To present the FE results in non-dimensional form, the value of Young's modulus is only chosen for convenience. The finite element model accounts for the appropriate loading and boundary conditions, as explained next.

\subsection{Loading and boundary condition}

One of the most important aspects of using the FEA is the selection and implementation of the most appropriate boundary conditions, which should lead to the average global behavior for the 2D FECVT models and avoid any localized deformation near the mesh boundaries. Three types of boundary conditions (BC) generally imposed by the FEA are the (1) periodic boundary condition, (2) prescribed displacement boundary condition, and (3) mixed boundary condition. Since the microstructures of the tissues (and the corresponding FECVT models) are not periodic, the periodic boundary condition is not appropriate. The prescribed displacement boundary condition is very strong restriction and is usually used with problems related to plastic deformation. The mixed boundary condition enforces the normal displacement, which eliminates the tangential force and the bending moments at the nodes on the boundaries. Since the mixed boundary condition tends to underestimate the Young's modulus (Zhu et al., 1997), the displacement boundary condition has been adopted here as it was proved to be appropriate for the analysis of non-periodic microstructures under uniaxial and biaxial loading (Fazekas et al., 2002; Shulmeister et al., 1998; Silva and Gibson, 1997; Silva et al., 1995; VanderBurg et al., 1997).



(a)

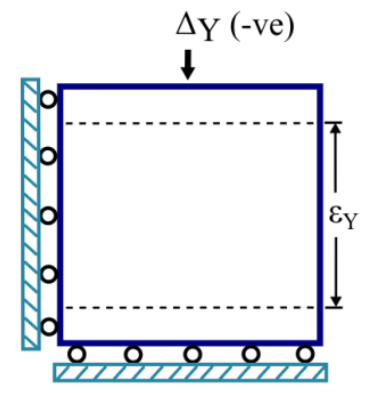

(b)



(c)

Fig.6. Simulated tests for determining effective stiffness properties. 
To determine the effective Young's modulus, the uniaxial compression load with prescribed displacement is simulated in both $X$ and $Y$ directions. A uniaxial compressive strain along the $X$ axis is imposed on the nodes of the right edge, while the nodes at the left edge are constrained from translating in the $\mathrm{X}$ direction as shown in Fig. 6. The nodes of the bottom edge also are constrained from translating in the $Y$ direction to prevent the rigid body motion. Similarly, uniaxial compression along $Y$ axis is also performed (Fig. 6[b]). In both cases, the nodes are constrained from rotating in the $X-Y$ plane. To determine the effective shear modulus (Fig. 6(c)), a biaxial loading test has been simulated with a positive displacement in the $X$ direction and a negative displacement in the $Y$ direction. The results have been computed for the alternative values of relative densities for the prescribed boundary condition.

\subsection{Determination of the effective stiffness properties}

For each model with a given relative density, the effective Young's modulus, $E^{*}$, and effective shear modulus, $G_{12}^{*}$, are determined. The macroscopic stress, $\sigma^{*}$, is calculated from the global reaction forces of the structure in the loading direction. The sum of the nodal reaction forces is divided by the edge length to determine the average normal stress in the loading direction. The strain, $\varepsilon^{*}$, is determined based on the technique of gage lines introduced by Silva (Silva et al., 1995) so as to eliminate the end effects. The displacement at each location, where the gage line intersects a cell wall, is computed by using a linear interpolation of the displacements of the two adjacent nodes. For a given pair of gage lines, the normal strain is computed as the change in distance between the gage lines divided by their original distance. The shear strain is computed as the change in the angle between the gage lines oriented at $45^{\circ}$ with respect to the coordinate axes. Nevertheless, the computed elastic constants for any model may vary by several percent, depending on the location of the gage lines.

\section{Results}

The effective stiffness of the collenchyma, parenchyma, and combined collenchymaparenchyma (col-par) tissues are predicted through the FEA of the FECVT models of the respective tissues. For each tissue, five FECVT models - the SVEs - have been considered based on the randomly chosen sections. The statistical results and the average effective stiffness properties are described in the following sub-sections. 


\subsection{Effective stiffness of collenchyma tissue using FEA}

The FECVT models of the collenchyma tissues are simulated in ANSYS to determine the effective tissue stiffness. Each of the FECVT models is simulated for a set of relative density, varying from $5 \%$ to $30 \%$. Fig. 7 shows the nodal displacements of the model tissue for $15 \%$ relative density, with compressive strain along $X$ and $Y$ directions. The microstructure in the $X$ direction is less stiff than that in the $Y$ direction. The microstructural anisotropy of the plant tissue originates from the cellular distribution.

Supplementary Figs. S.1(a) to S.1(c) in Appendix A, represent the variability of the computed stiffness with one standard error. The variations of the shear moduli are significant compared to the Young's moduli of the FECVT models at varying density.

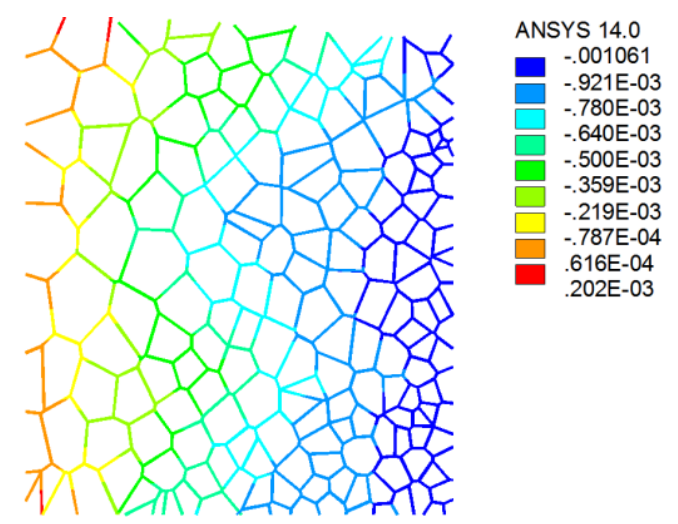

(a) Nodal displacement along $X$ direction

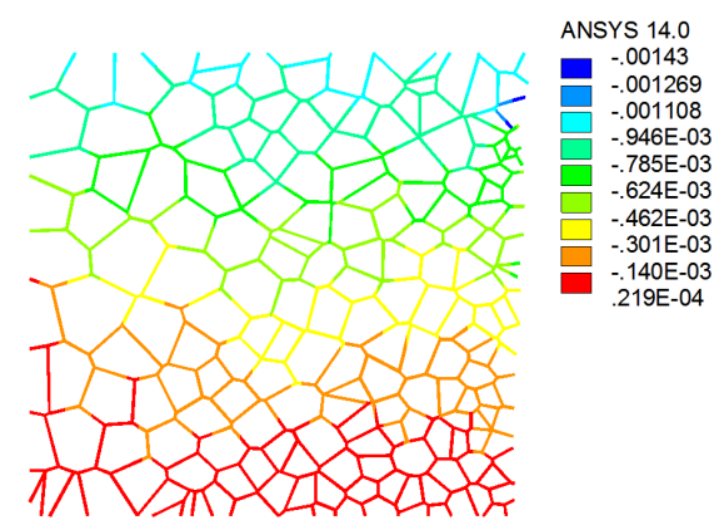

(b) Nodal displacement along $Y$ direction

Fig.7. Nodal displacement of the FECVT model of collenchyma tissue under uniaxial displacement BC. (a) $X$ component of nodal displacement and (b) $Y$ component of nodal displacement. 


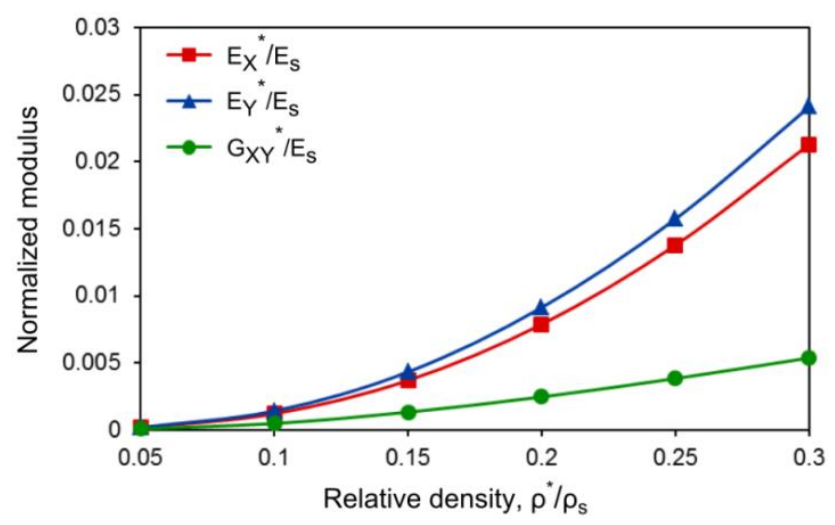

Fig.8. Normalized moduli of the FECVT model of collenchyma tissue.

The normalized effective stiffness of the FECVT model along the $Y$ direction is around $15 \%$ to $25 \%$ higher than that of the FECVT model along the $X$ direction for the range of density considered here (Fig. 8). The variation reflects the stiffening effect of the cell shape, size, and cellular distribution, and also depicts the anisotropic behavior of the collenchyma tissue of the rhubarb petiole. Supplementary Fig. S.2 (Appendix A) depicts the variability of the Poisson's ratio, $v_{X Y}^{*}$ and $v_{Y X}^{*}$, of the FECVT model of the collenchyma tissue. However, the effective Poisson's ratios, $v_{X Y}^{*}$ and $v_{Y X}^{*}$, exhibit no difference within the range of relative density (Fig. 9).

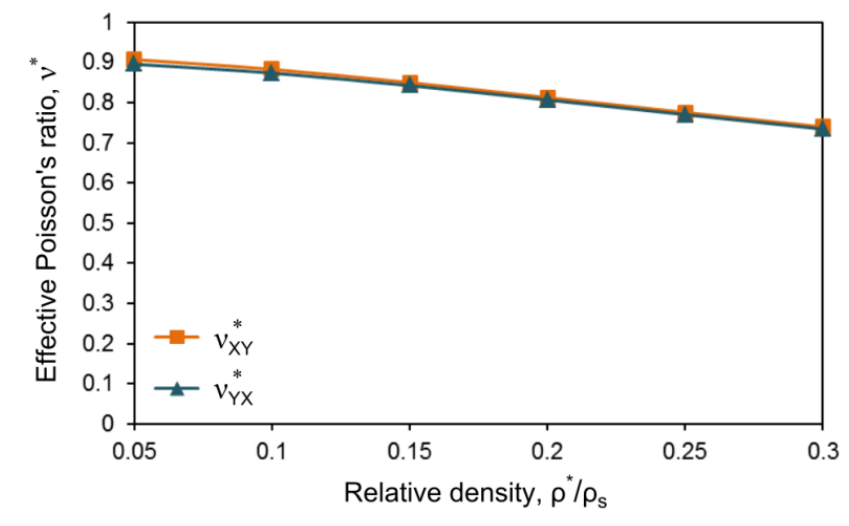

Fig.9. Effective Poisson's ratio of the FECVT model of collenchyma tissue.

\subsection{Apparent stiffness of parenchyma tissue using the FEA}

The FECVT models of the parenchyma sections also are simulated in ANSYS for the range of relative density, $0.05 \leq \rho^{*} / \rho_{s} \leq 0.30$, to determine the effective tissue stiffness. Fig. 10 shows the nodal displacements of model tissue for $15 \%$ relative density, with compressive strain 
along $X$ and $Y$ directions. The microstructure of the parenchyma tissue in the $Y$ direction is less stiff than that in the $X$ direction, whereas the collenchyma is less rigid in the $X$ direction.

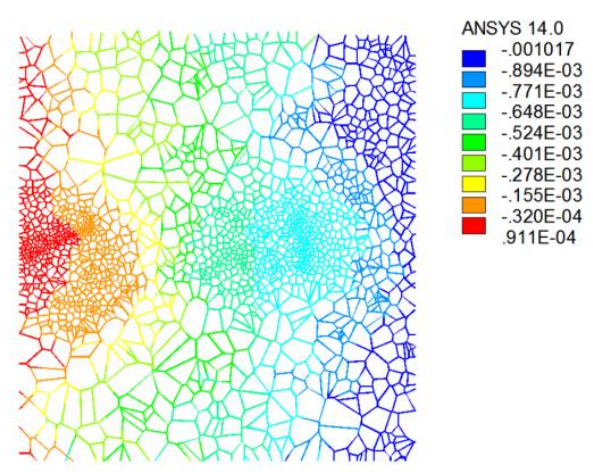

(a) Nodal displacement along $X$ direction

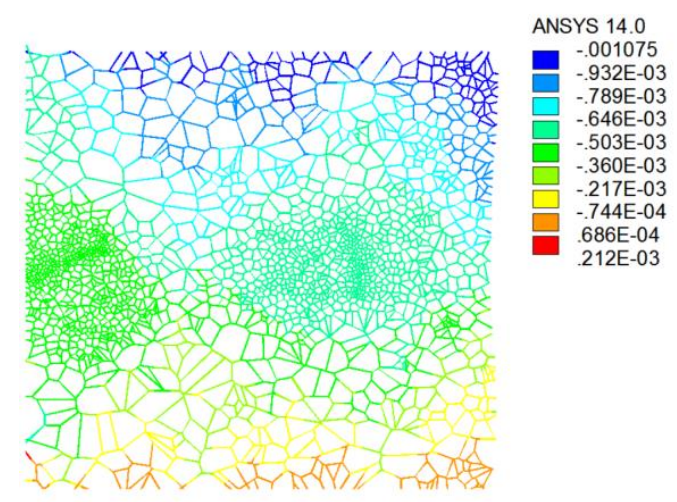

(b) Nodal displacement along $Y$ direction

Fig.10. Nodal displacement of the FECVT model of parenchyma tissue under uniaxial displacement BC: (a) $X$ component of nodal displacement, (b) $Y$ component of nodal displacement.

Supplementary Figs. S.3(a) to S.3(c) (Appendix A) represent the variability of the computed stiffness with one standard error. Similar to the collenchyma tissue, the variations of the shear moduli are significant compared with the Young's moduli of the FECVT models at varying relative density.

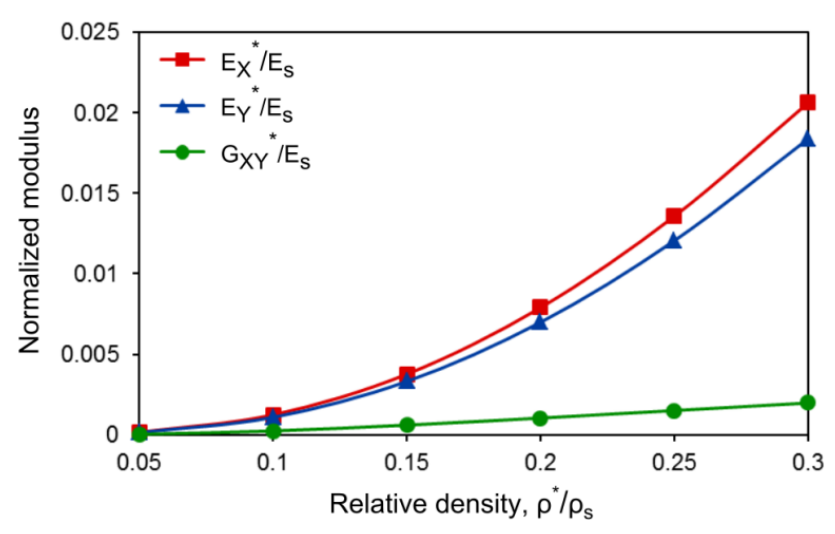

Fig.11. Normalized modulus of the FECVT model of parenchyma tissue.

The normalized effective stiffness of the parenchyma FECVT model along the $X$ direction is an average of $15 \%$ higher than that of the FECVT model along the $Y$ direction for 
the range of density considered here (Fig. 11). The clustered and graded cellularity of the vascular bundle stiffens the parenchyma tissue in $X$ direction compared to $Y$ direction. Along with the higher gradient in clustered regions, the cell size and cellular distribution lower the stiffness of the parenchyma tissue compared to the collenchyma tissue. Supplementary Fig. S.4 depicts the variability of the Poisson's ratios, $v_{X Y}^{*}$ and $v_{Y X}^{*}$, of the FECVT model of the parenchyma tissue. However, the effective Poisson's ratios, $v_{X Y}^{*}$ and $v_{Y X}^{*}$, of the FECVT model of the parenchyma tissue exhibit an average of $15 \%$ difference within the range of specified relative density (Fig. 12). From a structural point of view, the microarchitecture of the parenchyma tissue seems to be the origin of this variation.

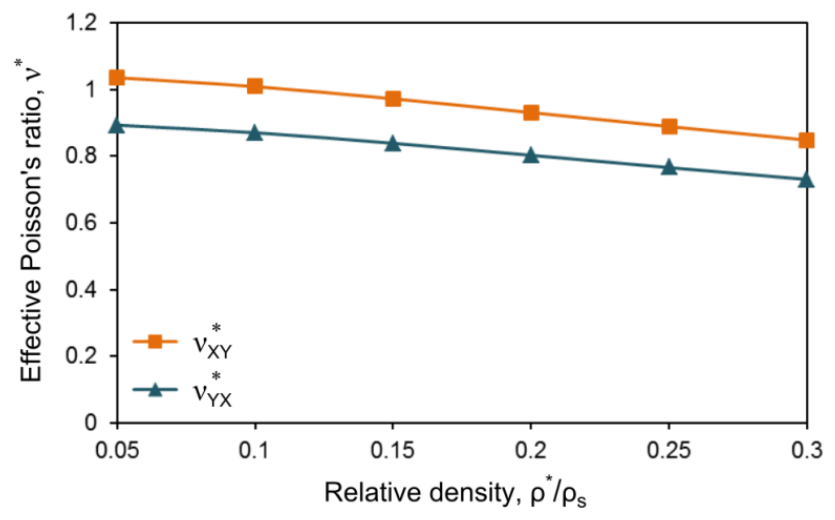

Fig.12. Effective Poisson's ratio of the FECVT model of parenchyma tissue.

\subsection{Apparent stiffness of collenchyma-parenchyma (Col-Par) tissue using the FEA}

Since the overall stiffness of a plant petiole or stem depends on the constituent tissues as a whole, the stiffness properties of the collective tissues also are analyzed in this section. To capture the cumulative and integrated effects of both the collenchyma and parenchyma (col-par) tissue, the FECVT models of the combined tissue sections are also simulated in ANSYS for the range of relative density, $0.05 \leq \rho^{*} / \rho_{s} \leq 0.30$, to determine the normalized tissue stiffness. Fig. 13 displays the nodal displacements of the col-par FECVT model for 15\% relative density, with compressive strain along the $X$ and $Y$ directions. The displacements along both directions display the same order of magnitude for the cellular distribution in the combined tissue. 


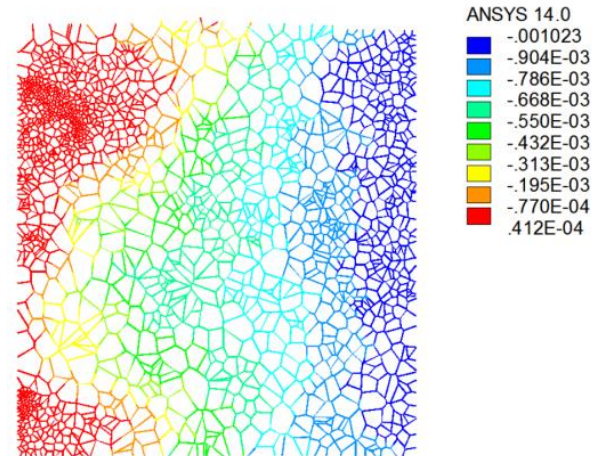

(b) Nodal displacement along $X$ direction

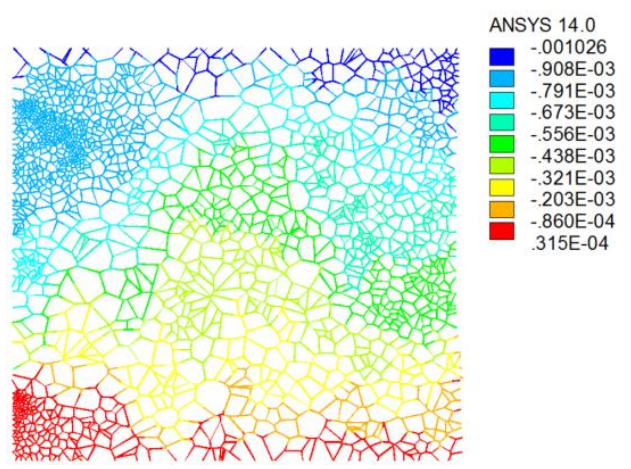

(b) Nodal displacement along $Y$ direction

Fig.13. Nodal displacement of the FECVT model of col-par tissue under uniaxial displacement BC: (a) $X$ component of nodal displacement, (b) $Y$ component of nodal displacement.

Supplementary Figs. S.5(a) to S.5(c) (Appendix A) represent the variability of the computed stiffness with one standard error. An expected trend of variability, similar to the collenchyma and parenchyma tissue, is observed for the combined tissues (Fig. 14). 


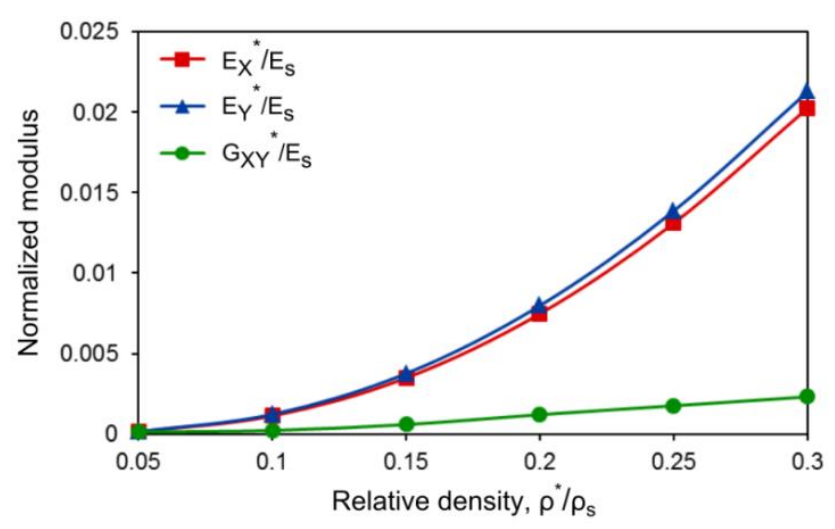

Fig.14. Normalized modulus of the FECVT model of collenchyma-parenchyma tissue.



Fig.15. Effective Poisson's ratio of the FECVT model of collenchyma-parenchyma tissue.

The combined effect of the collenchyma and parenchyma tissue is different than that of the individual tissues. The normalized effective stiffness of the col-par FECVT model is nearly similar (an average of 4\% higher along the $X$ direction) along both the $X$ and $Y$ directions throughout the relative density range considered here (Fig. 14). The overall effect of the combined tissues results in approximately equal stiffness. Nevertheless, the effective Young's moduli and shear modulus of the combined tissues are in-between the individual tissues. The Poisson's ratios, $v_{X Y}^{*}$ and $v_{Y X}^{*}$, of the FECVT model of the combined tissues also exhibit a difference between the ratios (Supplementary Fig. S.6 (Appendix A) and Fig. 15).

The normalized stiffness of the individual tissues and their combination provide insights into the effect of cell size, shape, and cellular distribution with clustered and complex area of 
higher gradients present in the rhubarb petiole. The analyses presented here manifest the micro architectural effect of constituent tissues.

\section{Discussion}

\subsection{Comparison of the normalized stiffness of various tissues}

The normalized stiffness of the FECVT models are compared to the stiffness of a randomly generated Voronoi model, and the stiffness calculated from closed-form expressions available in literature for periodic cellular materials and used for plant tissue modeling (Silva et al., 1995). Cellular periodicity, however, is an oversimplified assumption, not representative of the real random distribution of cells often seen in plant tissues. These formulas are obtained with an isotropic periodic cellular model that has a hexagon as a unit cell. On the other hand, the quasi-uniform random Voronoi model is generated for a set of uniformly distributed points. Supplementary Fig. S.7 (Appendix A) shows a regular hexagonal unit cell and the quasi-uniform randomly generated Voronoi model.

The normalized stiffness of the FECVT models of collenchyma, parenchyma, and combined collenchyma-parenchyma tissues; quasi-uniform Voronoi model; and the hexagonal unit cell are shown in Fig. 16. The FEA of the quasi-uniform random Voronoi model shows an average of $8 \%$ and $6 \%$ higher axial and shear modulus, respectively, compared to the closedform solutions obtained for the unit cell shown in Supplementary Fig. S.7(a) (Appendix A). The results of the moduli are in agreement with those presented by Gibson et al.(Gibson and Ashby, 1999; Silva et al., 1995). For a given set of relative density, the normalized effective elastic moduli of the different models at $X$ and $Y$ directions are shown in Figs. 16(a) and 16(b). With respect to relative density, $0.05 \leq \rho^{*} / \rho_{s} \leq 0.30$, the FECVT models of the rhubarb tissues exhibit nearly equal stiffness, which is $31 \%$ to $40 \%$ less rigid than the quasi-uniform random Voronoi model, and $29 \%$ to $35 \%$ less stiff than the periodic unit cell along the $X$ direction. On the other hand, the stiffness of the FECVT models of the rhubarb tissues varies along the $Y$ direction. Along this direction, for relative densities varying between $5 \%$ to $30 \%$, the collenchyma FECVT model is $20 \%$ to $39 \%$ less stiff than the random Voronoi model and $15 \%$ to $35 \%$ less rigid than the unit cell model; the parenchyma FECVT model is $22 \%$ to $47 \%$ more pliant than the random Voronoi model and $19 \%$ to $44 \%$ less stiff than the unit cell model. The 
col-par FECVT model is $18 \%$ to $39 \%$ more compliant than the quasi-uniform random Voronoi model and $24 \%$ to $33 \%$ than the unit cell model. Similarly, the shear moduli of the FECVT models are considerably lower than both the random Voronoi and unit cell models. The shear modulus of the collenchyma FECVT model is $30 \%$ to $57 \%$ less rigid than the quasi-uniform randomly generated Voronoi and hexagonal unit cell models. However, the FECVT models of the parenchyma and combined collenchyma-parenchyma tissues are much less stiff than the random Voronoi and the unit cell models.

To generate the Voronoi tessellation, a (quasi) uniform distribution of points has been imposed. Hence, the randomly generated Voronoi model displays a uniform cell size, a factor that influences the stiffness properties as shown in Fig. 16. In the FECVT model, both the shape and size of the cells, vary significantly with respect to the random Voronoi model and the hexagonal cell model. Therefore, the shape and size of the cells affect the normalized stiffness, which varies with density. The variation of the stiffness of the different FECVT models along the $X$ and $Y$ direction reflects the stiffening effect of the cell shape, size, and cellular distribution. Nonetheless, since the FECVT model contains smaller cells than those of the actual tissue, the stiffness could be overestimated. In addition, the short cell walls modeled by the beam element may impose spurious stiffness.

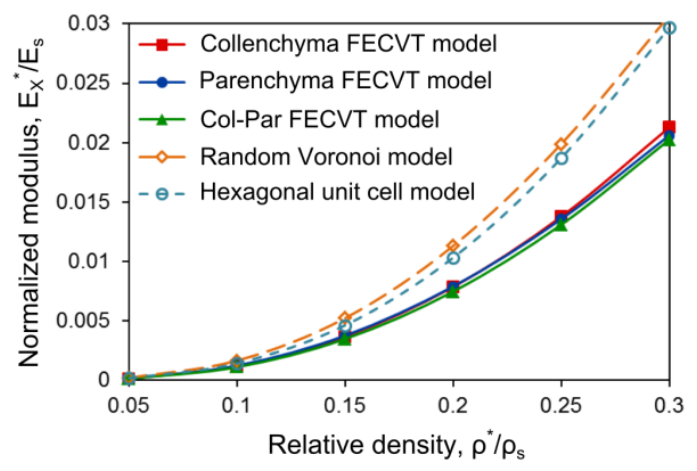

(a) Normalized modulus along $\mathrm{X}$-axis 


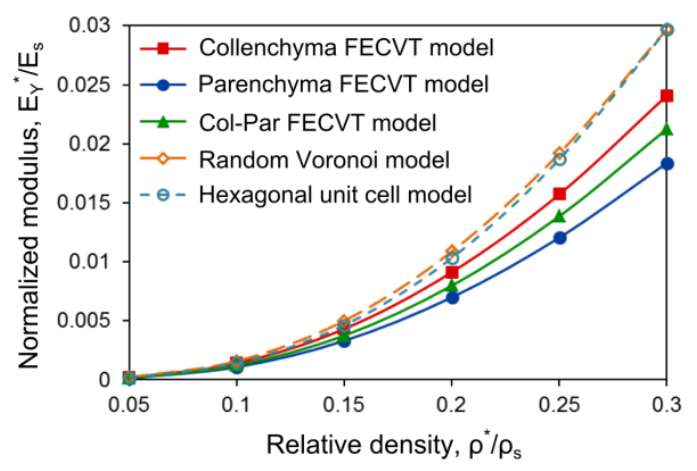

(b) Normalized modulus along y-axis

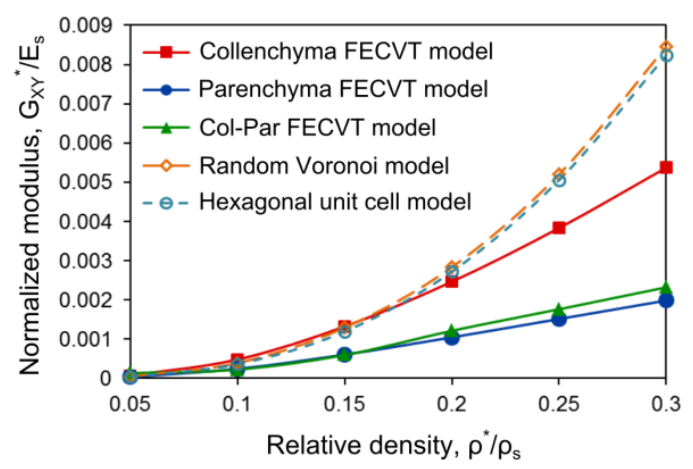

(c) Normalized shear modulus

Fig.16. Comparison of the normalized modulus of the FECVT models of the different constituent tissues to quasi-uniform random Voronoi and hexagonal unit cell models.

Fig. 17 depicts the effective Poisson's ratios for the FECVT, random Voronoi, and hexagonal unit cell models. The finite element analyses of the different FECVT models and random Voronoi models exhibit a marginal difference between the Poisson's ratio of $v_{X Y}^{*}$ and $v_{Y X}^{*}$. The Poisson's ratios of the FECVT, and the random Voronoi model are weakly dependent on relative density, and the microarchitecture of the tissue weakly affects the Poisson's ratio. 


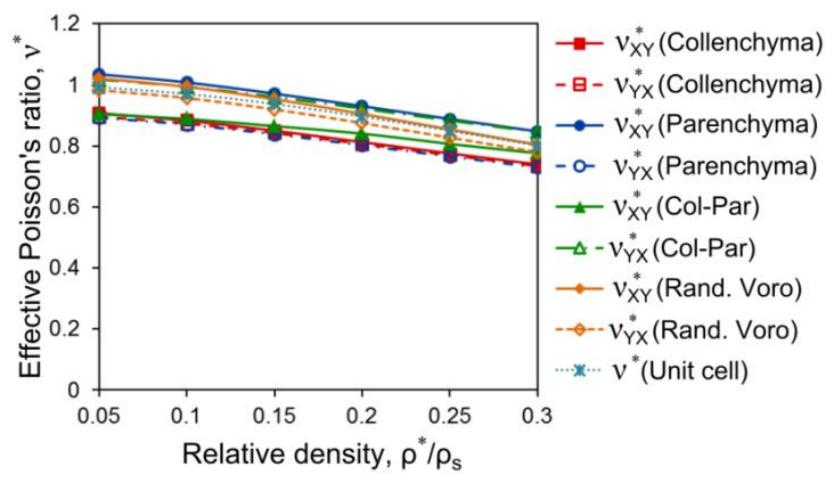

Fig.17. Comparison of effective Poisson's ratio of the FECVT models of the different constituent tissues to quasi-uniform random Voronoi and hexagonal unit cell models.

The effective stiffness of the cellular tissue can be obtained from the computed wall stiffness (Faisal et al., 2013; Faisal, 2013). The axial stiffness of parenchyma tissue lies between 13.2 and 19.6 MPa and the shear modulus is approximately 6.7 MPa. The experimental stiffness of fresh parenchyma tissue was obtained from compression tests of cubes of parenchyma immersed in a solution of $0.02 \mathrm{M}$ each of potassium phosphate monobasic and potassium phosphate dibasic (N. Hristozov, unpublished data). The experimental stiffness of the fresh parenchyma tissue is approximately $5 \mathrm{MPa}$, which is considerably lower than the computed stiffness of the respective tissue. This difference may result from experimental and biological factors as well as from the computer model as it currently stands. First, the orientation of the parenchyma cubes during the compressive tests differed from that used in the computational model (longitudinal in the experiment versus transverse in the model). In addition to the orientation of the compressive force, the osmotic strength of the buffer used during testing can affect the experimental results since the internal turgor pressure within the fluid-filled cells plays an important role in controlling the cellular stiffness. Ideally, this effect would be quantified and included in the model. Another biological factor that can play a role is the chemical make-up and molecular organization of the cell walls, which can differ between cells and cell types (e.g. the vascular tissue is reinforced by the production of internal secondary cell walls; dissimilar cellulose microfibril angles can alter wall strength in specific directions). In the computational model, the tissue stiffness is derived from the wall stiffness based on an average of $30 \%$ cellulose microfibrils, $60 \%$ pectin, and $10 \%$ hemicelluloses - a typical primary cell wall configuration for dicotyledonous plants (Faisal, 2013). Hence, instead of using the generic 
composition of the dicotyledonous plant cell wall, the cell wall composition and the microfibril angle (MFA) of the model plant should be determined via experiments. This would enable the corresponding computational stiffness to provide a more realistic prediction of the actual tissue stiffness. Finally, as noted earlier, the FECVT model can result in a smaller cell size per unit area, thereby resulting in higher numbers of both cells and cell walls, factors that would increase the stiffness of the model. These factors reflect the complexity of plant tissue systems and the need to consider multiple parameters during their modeling. Nevertheless, the utility of our system is demonstrated and the computational stiffness derived using values from the literature provides theoretical bounds for the stiffness of the constituent tissues. This step is essential to develop a multiscale model capable of predicting the overall mechanical properties of plant petioles and stems from the structural properties obtained at each length scale as well as the properties of the constituent tissues.

\section{Conclusion}

Cellular solids are prevalent both in nature and in human-made engineering structures. Their constituent materials and the way they are architectured across the length scale govern the mechanical response at the macroscale. A micromechanical analysis, capturing the realistic arrangement of the microstructure can thus provide insight into the overall macroscale behavior of a cellular material. The FECVT method is general and not tailored for a specific species of plant tissue. It has already been applied to reproduce the tissue geometry of Arabidopsis thaliana and Philodendron melinonni (Faisal et al., 2012). Furthermore, FEA has been carried out to predict the mechanical properties of the surrogated models describing the tissue of $P$. melinonii (Faisal et al., 2011). This paper has presented a microstructural analysis of rhubarb tissues, which can be considered as a paradigm of random cellular structures with complex heterogeneity. The geometric models developed via the FECVT algorithm surrogate the constituent tissues of the rhubarb petiole, thereby allowing the calculation of the elastic properties of a cellular tissue. Finite element analysis has been used as a computational mean to assess the constituent tissue properties of the rhubarb petiole, whose cellular microstructures have been generated by the FECVT algorithm. Instead of a large scale RVE, the statistical volume elements of the tissue are considered to capture the microstructural irregularity present in the tissue and compute its stiffness via FEA. The finite element analysis of the SVEs depicts the 
impact of complex heterogeneity and graded cellularity in the tissues. The cell shape, size, and cellular distribution of the collenchyma and parenchyma tissues are shown to have a different impact on their respective normalized stiffness properties. The collenchyma tissue is found to be stiff along the $Y$ direction, whereas the parenchyma tissue stiff in the $X$ direction. The variation of stiffness along the $X$ and $Y$ directions are due to the heterogeneous cellularity and clustered higher area gradients observed in the parenchyma tissue, albeit the directions are chosen arbitrarily. The axial stiffness of the combined tissue, on the other hand, is similar in both directions. The shear modulus of the collenchyma tissue has been also found higher compared to the parenchyma tissue, and in-between for the tissues as a whole. By comparing these results with the quasi-uniform randomly generated Voronoi and hexagonal unit cell models, a clear inference can be made about the nonhomogeneous cellularity in the rhubarb tissues, a factor that make them more compliant than the predictions obtained with the periodic as well as quasiuniform random cellular microstructure.

\section{Acknowledgement}

This research is supported by a grant from Fonds de Recherche du Québec-Nature et Technologies (FQRNT).

\section{Conflict of Interest}

The authors declare no conflict of interest. 


\section{References}

Alber, I., Bassani, J.L., Khantha, M., Vitek, V., Wang, G.J., 1992. Grain Boundaries as Heterogeneous Systems: Atomic and Continuum Elastic Properties. Philosophical Transactions of the Royal Society of London. Series A: Physical and Engineering Sciences 339, 555-586.

Barber, C.B., Dobkin, D.P., Huhdanpaa, H., 1996. The Quickhull algorithm for convex hulls. Acm Transactions on Mathematical Software 22, 469-483.

Beakou, A., Ntenga, R., 2011. Structure, morphology and mechanical properties of Rhectophyllum camerunense (RC) plant fiber. Part II: Computational homogenization of the anisotropic elastic properties. Computational Materials Science 50, 1550-1558.

Cailletaud, G., Forest, S., Jeulin, D., Feyel, F., Galliet, I., Mounoury, V., Quilici, S., 2003. Some elements of microstructural mechanics. Computational Materials Science 27, 351-374.

Canny, J., 1986. A computational approach to edge detection. IEEE Transaction of Pattern Analysis and Machine Intelligence PAMI-8, 679-698.

Chan, P.K., Rey, A.D., 1997. Polymerization-Induced Phase Separation. 2. Morphological Analysis. Macromolecules 30, 2135-2143.

Faisal, T., Rey, A., Pasini, D., 2013. A Multiscale Mechanical Model for Plant Tissue Stiffness. Polymers 5, 730-750.

Faisal, T.R., 2013. A multiscale approach to mechanical modeling of a leaf petiole: Integrating cell wall, cellular tissues and structural morphology, McGill University, Montreal, QC, Canada.

Faisal, T.R., Rey, A.D., Pasini, D. 2011. Hierarchical Microstructure and Elastic Properties of Leaf Petiole Tissue in Philodendron melinonii MRS Vol. 1420. Cambridge University Press.

Faisal, T.R., Khalil Abad, E.M., Hristozov, N., Pasini, D., 2010. The Impact of Tissue Morphology, CrossSection and Turgor Pressure on the Mechanical Properties of the Leaf Petiole in Plants. Journal of Bionic Engineering 7, Supplement, S11-S23.

Faisal, T.R., Hristozov, N., Rey, A.D., Western, T.L., Pasini, D., 2012. Experimental determination of Philodendron melinonii and Arabidopsis thaliana tissue microstructure and geometric modeling via finite-edge centroidal Voronoi tessellation. Physical Review E 86, 031921.

Fazekas, A., Dendievel, R., Salvo, L., Brechet, Y., 2002. Effect of microstructural topology upon the stiffness and strength of 2D cellular structures. International Journal of Mechanical Sciences 44, 2047-2066.

Ghosh, S., Lee, K., Moorthy, S., 1996. Two scale analysis of heterogeneous elastic-plastic materials with asymptotic homogenization and Voronoi cell finite element model. Computer Methods in Applied Mechanics and Engineering 132, 63-116.

Gibson, L.J., Ashby, M.F., 1982. The mechanics of 3-dimensional cellular materials. Proc. R. Soc. London Ser. A-Math. Phys. Eng. Sci. 382, 43-59.

Gibson, L.J., Ashby, M.F., 1999. Cellular solids : structure and properties. $2^{\text {nd }}$ ed. Cambridge University Press, Cambridge ; New York.

Huang, T., Tsuji, T., Kamal, M.R., Rey, A.D., 1999. Structural characterization of grain pattern diversity in parametric space. Journal of Materials Science 34, 4551-4561.

Hussain, K., De los Rios, E.R., Navarro, A., 1993. A two-stage micromechanics model for short fatigue cracks. Engineering Fracture Mechanics 44, 425-436.

Kamal, M.R., Huang, T., Rey, A.D., 1997. Topological properties of polymer spherulitic grain patterns from simultaneous nucleation. Journal of Materials Science 32, 4085-4099.

Li, K., Gao, X.L., Subhash, G., 2005. Effects of cell shape and cell wall thickness variations on the elastic properties of two-dimensional cellular solids. International Journal of Solids and Structures 42, 1777-1795. 
Liu, W.K., Qian, D., Gonella, S., Li, S., Chen, W., Chirputkar, S., 2010. Multiscale methods for mechanical science of complex materials: Bridging from quantum to stochastic multiresolution continuum. International Journal for Numerical Methods in Engineering 83, 1039-1080.

Mattea, M., Urbicain, M.J., Rotstein, E., 1989. Computer model of shrinkage and deformation of cellular tissue during dehydration. Chemical Engineering Science 44, 2853-2859.

Nemat-Nasser, S., Hori, M., 1999. Micromechanics: overall properties of heterogeneous materials Elsevier Amsterdam.

Ntenga, R., Beakou, A., 2011. Structure, morphology and mechanical properties of Rhectophyllum camerunense (RC) plant-fiber. Part I: Statistical description and image-based reconstruction of the cross-section. Computational Materials Science 50, 1442-1449.

Ostoja-Starzewski, M., 1993. Micromechanics as a basis of random elastic continuum approximations. Probabilistic Engineering Mechanics 8, 107-114.

Ostoja-Starzewski, M., 1998. Random field models of heterogeneous materials. International Journal of Solids and Structures 35, 2429-2455.

Ostoja-Starzewski, M., 2006. Material spatial randomness: From statistical to representative volume element. Probabilistic Engineering Mechanics 21, 112-132.

Pasini, D., 2008. On the biological shape of the Polygonaceae Rheum rhabarbarum petiole. Journal of Design \& Nature and Ecodynamics 3, 25.

Roberts, A.P., Garboczi, E.J., 2001. Elastic moduli of model random three-dimensional closed-cell cellular solids. Acta Materialia 49, 189-197.

Roudot, A.C., Duprat F., Pietri E., 1990. Simulation of a penetrometric test on apples using VoronoiDelaunay tessellation. Food structure 9, 215-222.

Shulmeister, V., Van der Burg, M.W.D., Van der Giessen, E., Marissen, R., 1998. A numerical study of large deformations of low-density elastomeric open-cell foams. Mechanics of Materials 30, 125140.

Silva, M.J., Gibson, L.J., 1997. The effects of non-periodic microstructure and defects on the compressive strength of two-dimensional cellular solids. International Journal of Mechanical Sciences 39, 549-563.

Silva, M.J., Hayes, W.C., Gibson, L.J., 1995. The effects of nonperiodic microstructure on the elastic properties of 2-dimensional cellular solids. International Journal of Mechanical Sciences 37, 1161-1177.

VanderBurg, M.W.D., Shulmeister, V., VanderGeissen, E., Marissen, R., 1997. On the linear elastic properties of regular and random open-cell foam models. Journal of Cellular Plastics 33, 31-\&.

Yin, X., Chen, W., To, A., McVeigh, C., Liu, W.K., 2008. Statistical volume element method for predicting microstructure-constitutive property relations. Computer Methods in Applied Mechanics and Engineering 197, 3516-3529.

Zhu, H.X., Knott, J.F., Mills, N.J., 1997. Analysis of the elastic properties of open-cell foams with tetrakaidecahedral cells. Journal of the Mechanics and Physics of Solids 45, 319-343. 


\section{Supplementary Figures:}

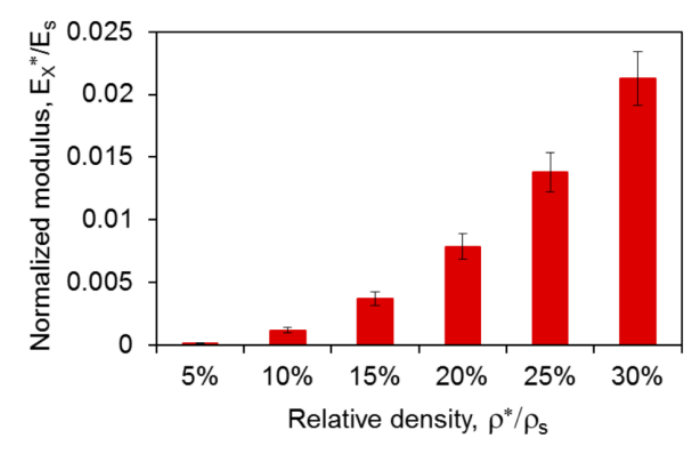

(a) Young's modulus along $X$ - axis

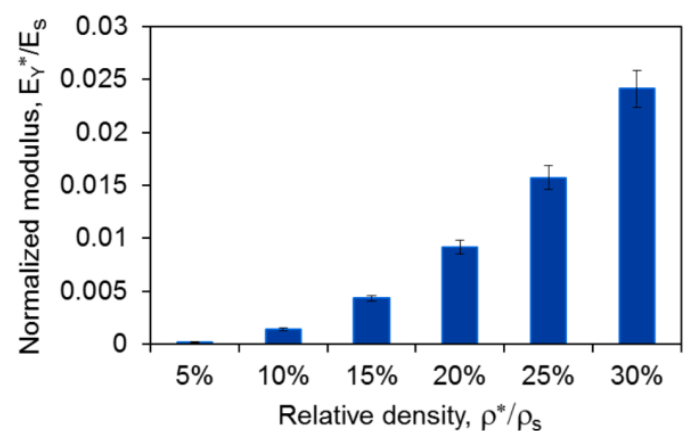

(b) Young's modulus along $Y-$ axis

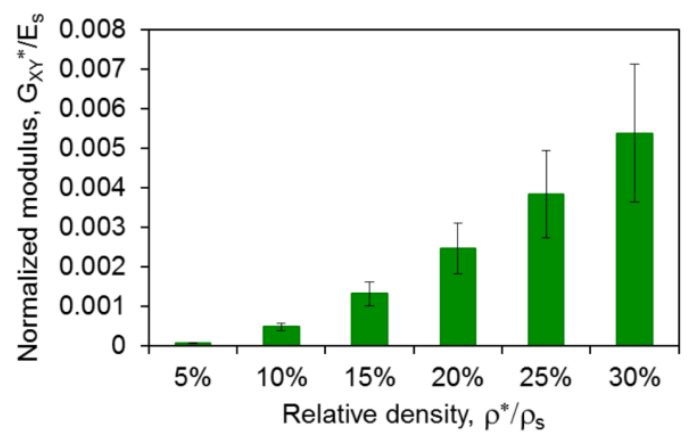

(c) Shear modulus

Fig.S.1. Variablity of the mean stiffness of the FECVT models of collenchyma tissue for varying relative density with one standard error.

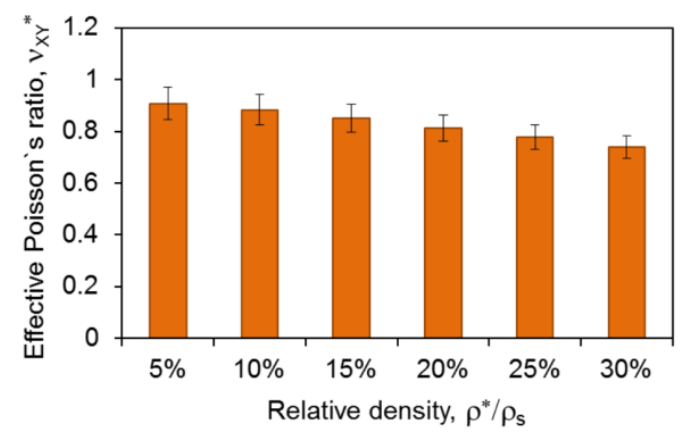

ratio, $v_{X Y}^{*}$

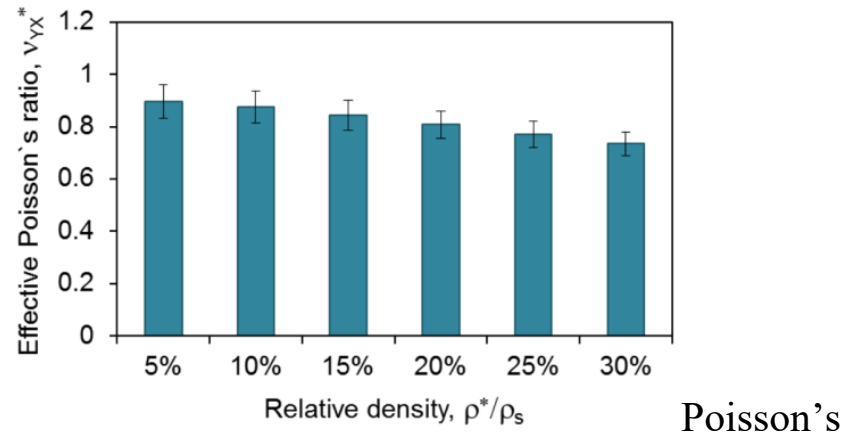

(b) Poisson's ratio, $v_{Y X}^{*}$ 
Fig.S.2. Variablity of mean Poisson's ratio of the FECVT models of collenchyma tissue for varying relative density with one standard error.

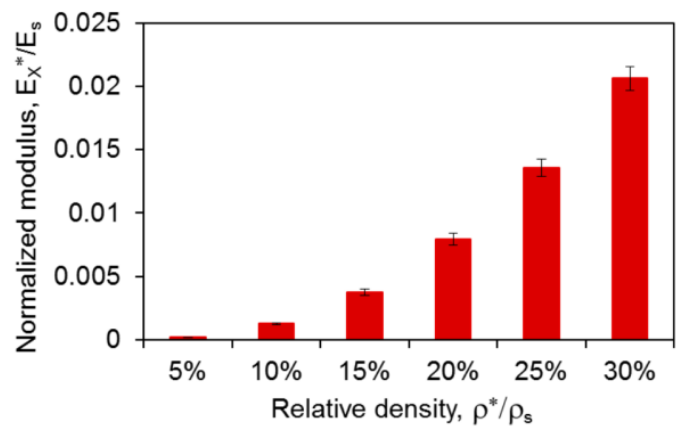

(a) Young's modulus along $X$-axis

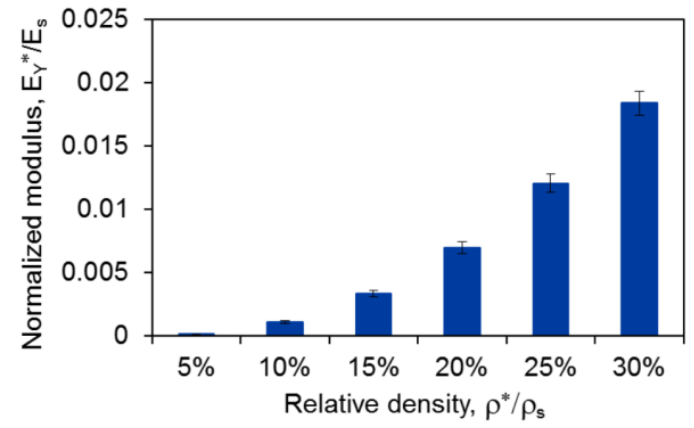

(b) Young's modulus along $Y$-axis

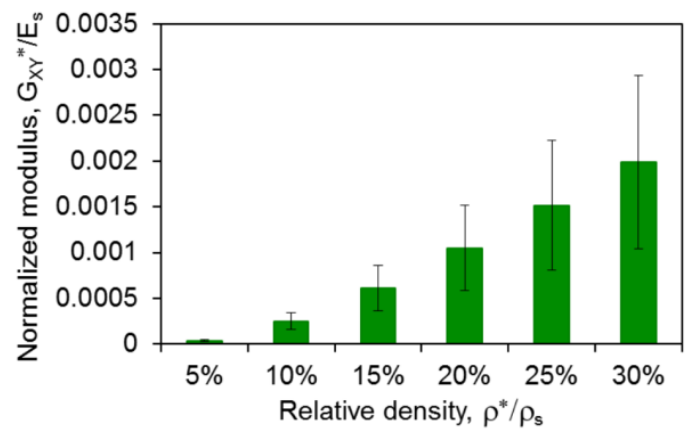

(c) Shear modulus

Fig.S.3. Variablity of mean stiffness of the FECVT models of the parenchyma tissue for varying relative density with one standard error.

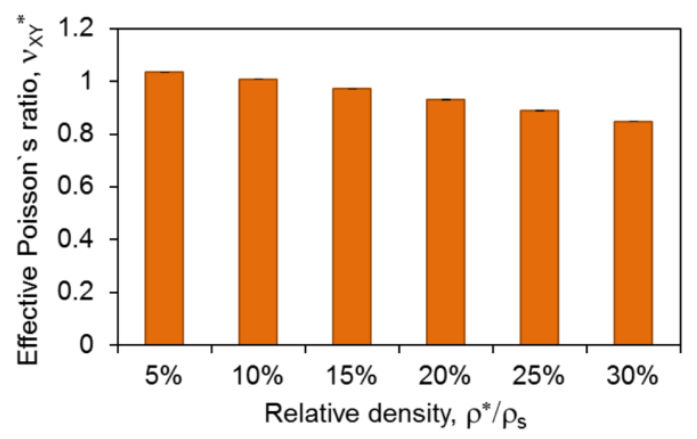

(a) Major Poisson's ratio

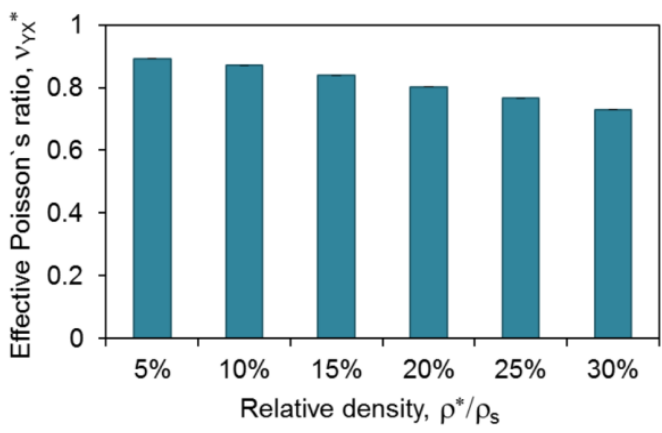

(b) Minor Poisson's ratio 
Fig.S.4. Variablity of mean Poisson's ratio of the FECVT models of parenchyma tissue for varying relative density with one standard error.



(a) Young's modulus along $X$-axis

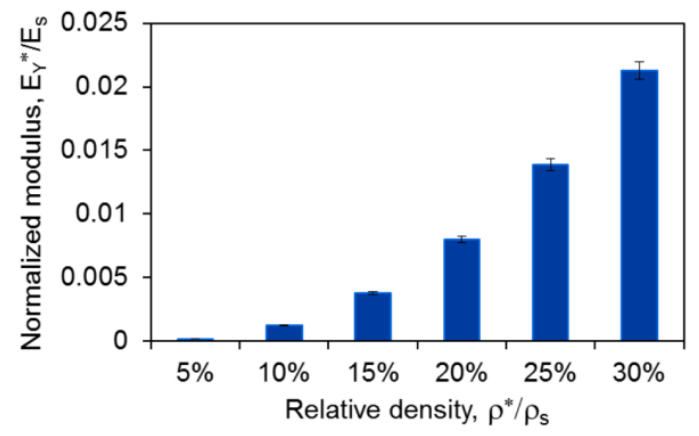

(b) Young's modulus along $Y$ - axis

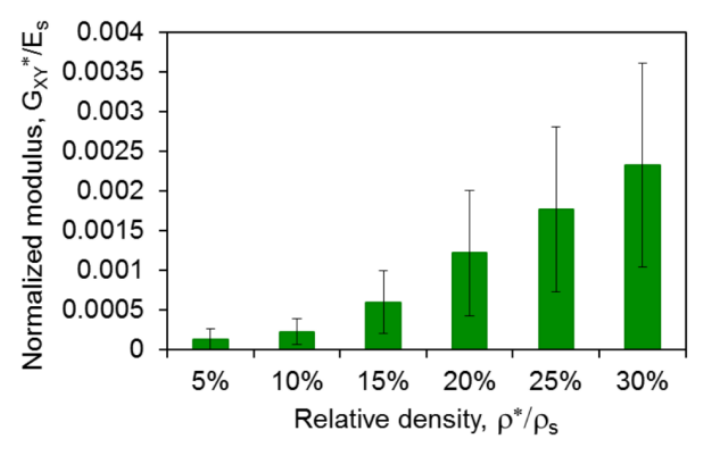

(c) Shear modulus

Fig.S.5. Variablity of mean stiffness of the FECVT models of combined collenchymaparenchyma tissue for varying relative density with one standard error.

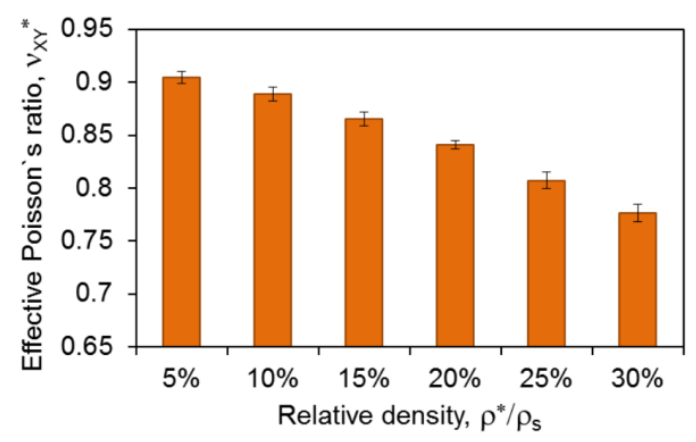

(a) Major Poisson's ratio

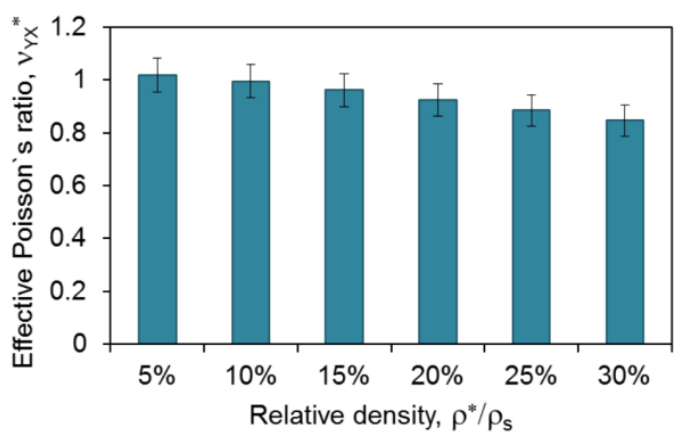

(b) Mionr Poisson's ratio 
Fig.S.6. Variablity of mean Poisson's ratio of the FECVT model of collenchyma-parenchyma tissue for varying relative denisty with one standard error.



(a)

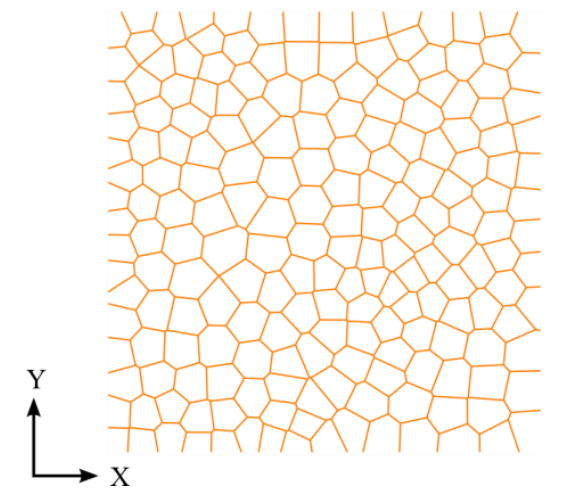

(b)

Fig.S.7. (a) Hexagonal unit cell (b) Quasi-uniform random Voronoi model. 


\section{List of Figures}

Fig.1. Multiscale hierarchical organization of a plant (Rheum rhabarbarum) petiole. [n] denotes the hierarchical level of the multiscale structure.

Fig.2. Paraffin-embedded rhubarb petiole cross-section stained with TBO and imaged with light microscopy at 20x magnifications. Approximately 16 photos were stitched together to create this composite image. Collenchyma cells, vascular bundle, and parenchyma cells are visible. Scale bar $=50 \mu \mathrm{m}$.

Fig.3. FECVT model of collenchyma tissue of rhubarb petiole.

Fig.4. FECVT model of parenchyma tissue of rhubarb petiole.

Fig.5. FECVT model of combined collenchyma-parenchyma (col-par) tissue of rhubarb petiole.

Fig.6. Simulated tests for determining effective stiffness properties.

Fig.7. Nodal displacement of the FECVT model of collenchyma tissue under uniaxial displacement BC. (a) $X$ component of nodal displacement and (b) $Y$ component of nodal displacement.

Fig.8. Normalized moduli of the FECVT model of collenchyma tissue.

Fig.9. Effective Poisson's ratio of the FECVT model of collenchyma tissue.

Fig.10. Nodal displacement of the FECVT model of parenchyma tissue under uniaxial displacement BC: (a) $X$ component of nodal displacement, (b) $Y$ component of nodal displacement.

Fig.11. Normalized modulus of the FECVT model of parenchyma tissue.

Fig.12. Effective Poisson's ratio of the FECVT model of parenchyma tissue.

Fig.13. Nodal displacement of the FECVT model of col-par tissue under uniaxial displacement BC: (a) $X$ component of nodal displacement, (b) $Y$ component of nodal displacement.

Fig.14. Normalized modulus of the FECVT model of collenchyma-parenchyma tissue.

Fig.15. Effective Poisson's ratio of the FECVT model of collenchyma-parenchyma tissue. 
Fig.16. Comparison of the normalized modulus of the FECVT models of the different constituent tissues to quasi-uniform random Voronoi and hexagonal unit cell models.

Fig.17. Comparison of effective Poisson's ratio of the FECVT models of the different constituent tissues to quasi-uniform random Voronoi and hexagonal unit cell models.

Fig.S.1. Variablity of the mean stiffness of the FECVT models of collenchyma tissue for varying relative density with one standard error.

Fig.S.2. Variablity of mean Poisson's ratio of the FECVT models of collenchyma tissue for varying relative density with one standard error.

Fig.S.3. Variablity of mean stiffness of the FECVT models of the parenchyma tissue for varying relative density with one standard error.

Fig.S.4. Variablity of mean Poisson's ratio of the FECVT models of parenchyma tissue for varying relative density with one standard error.

Fig.S.5. Variablity of mean stiffness of the FECVT models of combined collenchymaparenchyma tissue for varying relative density with one standard error.

Fig.S.6. Variablity of mean Poisson's ratio of the FECVT model of collenchyma-parenchyma tissue for varying relative denisty with one standard error.

Fig.S.7. (a) Hexagonal unit cell (b) Quasi-uniform random Voronoi model. 
